\title{
Laparoscopic cholecystectomy as a teaching operation: comparison of outcome between residents and attending surgeons in 1,747 patients
}

\author{
René Fahrner • Matthias Turina $\cdot$ Valentin Neuhaus • \\ Othmar Schöb
}

Received: 25 March 2011 / Accepted: 10 October 2011 /Published online: 20 October 2011

(C) Springer-Verlag 2011

\begin{abstract}
Purpose Standardized surgical training is increasingly confronted with the public demand for high quality of surgical care in modern teaching hospitals. The aim of this study was to compare the results of laparoscopic cholecystectomy (LC) performed by resident surgeons (RS) and attending surgeons (AS).

Methods In this retrospective review of prospectively collected data $1,747 \mathrm{LC}$ were performed in a community hospital between 1999 and 2009. Seven hundred seventy operations were performed by RS. Parameters analysed included the duration of operation and length of hospital stay, intraoperative complications, 30-day morbidity and mortality.

Results Duration of operation was 88 (25-245) min for RS vs. $75(30-190)$ min by AS ( $p=0.001)$. Elective operations were shorter when performed by AS (70 (30-190) [AS] vs. 85 (25-240) [RS] min, $p=0.001)$. Length of hospital stay was shorter in patients treated by RS (4 (1-49) days [RS] vs. 5 (1-83) days [AS], $p=0.1)$. Intraoperative complica-
\end{abstract}

R. Fahrner $\cdot$ M. Turina $\cdot$ V. Neuhaus $\cdot$ O. Schöb

Department of Surgery, Spital Limmattal,

8952 Schlieren, Switzerland

R. Fahrner $(\square)$

Division of Visceral Surgery and Medicine,

University Hospital of Bern,

3010 Bern, Switzerland

e-mail: r.fahrner@web.de

M. Turina

Department of Surgery, Stadtspital Triemli,

8063 Zürich, Switzerland

V. Neuhaus

Department of Surgery, University Hospital of Zürich,

8091 Zürich, Switzerland tions showed no differences between the groups $(1.0 \%$ [RS] vs. $1.3 \%$ [AS], $p=0.6$ ), whereas 30 -day morbidity was lower in patients treated by RS $(3.8 \%[\mathrm{RS}]$ vs. $6.2 \%$ [AS], $p=0.02$ ). Overall mortality was $0.6 \%$ and independent of surgical expertise $(0.5 \%$ [RS] vs. $0.8 \%$ [AS], $p=0.5)$.

Conclusions Provided adequate training, supervision and patient selection, surgical residents are able to perform LC with results comparable to those of experienced surgeons.

Keywords Laparoscopic cholecystectomy · Teaching operation · Complications · Quality control · Outcome

\section{Introduction}

Since the first laparoscopic cholecystectomy (LC) was performed in the late 1980s [1], this procedure has become the treatment of choice in symptomatic cholelithiasis as well as in acute and chronic cholecystitis [2]. Because of the good results, the public interest has increased considerably over the following years [3]. This demand has led to an increased pressure on young surgeons to learn laparoscopic skills in the early stage of their surgical training [4].

Laparoscopic surgery is technically more demanding than open procedures and requires good three-dimensional perception skills. In addition, tissue handling in laparoscopic surgery is different to open procedures, as organs (e.g. intestine, gallbladder, liver) by nature cannot be felt directly. In contrast to open procedures, laparoscopic operations are often performed by experienced surgeons, therefore young surgeons may experience a delay in their surgical training. Resident surgeons (RS) need a structured surgical training with appropriate instruction of an attending surgeon (AS) during the surgical procedures to avoid complications during the learning curve [5]. 
Standardized, efficient surgical training is increasingly confronted with the public demand for high quality and cost effectiveness of surgical care in modern teaching hospitals [6]. This leads to a controversy in teaching hospitals to overcome the problem of high surgical quality on the one hand and the necessity of education of surgical trainees [7] on the other hand. Previous studies have shown that operations performed by resident surgeons were associated with higher costs $[8,9]$ but without higher perioperative morbidity for patients treated by them $[8,10-12]$.

In this study we have chosen to analyse the LC as a frequently performed laparoscopic procedure which is nowadays part of the basic surgical training in many institutions. Our study is one of the largest single-centre investigations on LC concerning teaching issues in Europe. Residents in their third year started to perform LC in supervision of an attending surgeon. All operations were based on strict internal guidelines and routine use of intraoperative cholangiography. The aim of our present study was to analyse whether LC as a standardized teaching operation is safe in the hands of RS when compared to AS with respect to general and specific operative morbidity, mortality, duration of operation and length of hospital stay.

\section{Material and methods}

\section{Clinical setting}

The study was performed in a 300-bed community hospital in an urban environment of about 1.5 million inhabitants. Between January 1, 1999 and December 31, 2009, 1,747 LC were performed because of biliary colic, acute or chronic cholecystitis. All operations were started as laparoscopic procedures. Strictly defined surgical training residents with at least 2 years of surgical practice performed LC and were all assisted by AS to provide the highest level of patient's safety.

\section{Operative technique}

The operative technique was performed in a standardized manner. Creation of the pneumoperitoneum was performed using the Veress needle, and in total, four trocars were inserted. Initially preparation of the triangle of Calot was performed from postero-lateral to reach the "critical view of safety" to identify and dissect the cystic duct and cystic artery. After clipping the cystic duct distally, intraoperative cholangiography was performed routinely (Fig. 1a, b). In case of bile duct concrements, the further operative steps were dependent on the findings: concrements near the cystic duct were attempted to be
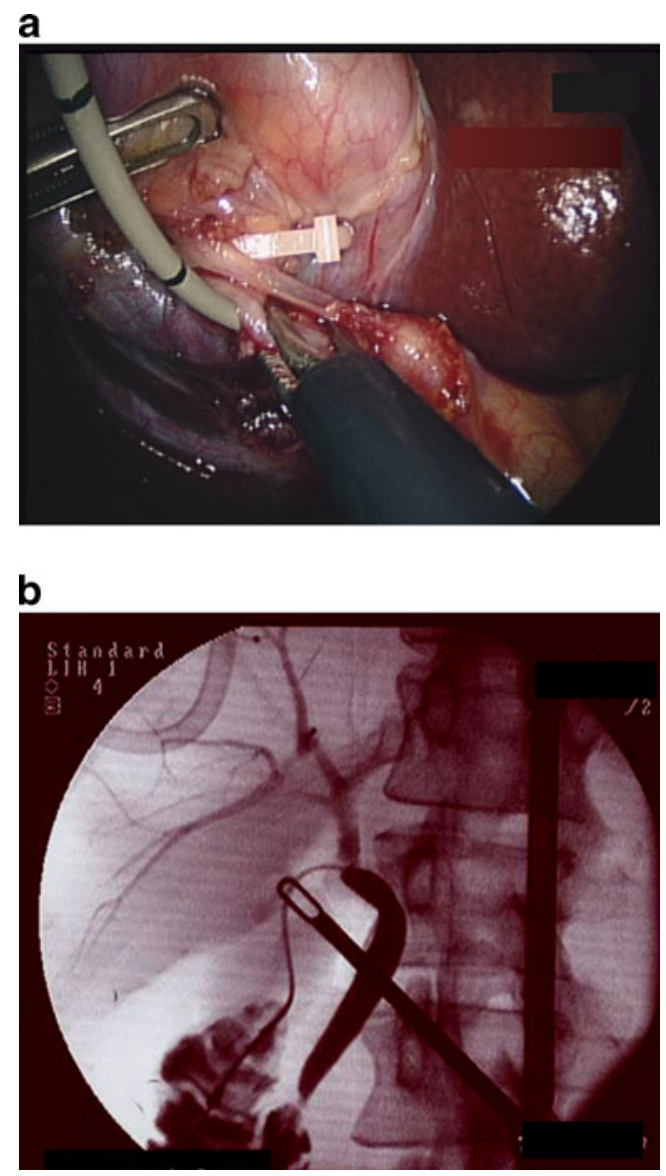

Fig. 1 a Intraoperative situs with an inserted catheter in the opened cystic duct for intraoperative cholangiography. b X-ray picture of intraoperative cholangiography with identification of the cystic duct, main hepatic branches and common bile duct without concrements or strictures

removed by a Fogarty manoeuvre. Alternatively, endoscopic retrograde cholangiopancreatography was performed postoperatively to remove residual stones. After removing the catheter, the cystic duct was clipped twice and the gallbladder was dissected of the liver bed after clipping and dissecting the cystic artery and finally removed by using an endobag.

\section{Data collection}

This retrospective analysis was based on a prospectively led clinical database of the AQC (Swiss quality working group, [13]), a voluntary quality working group of Swiss surgical departments. All data are entered in a centralized database (Qualicare; Qualidoc, Liebefeld-Bern, Switzerland) and are analysed by each surgical department.

All data were obtained and analysed according to the University of Zürich Institutional Review Board guidelines and in strict adherence to the ethical guidelines for human research of the Swiss Academy of Medical Sciences (Schweizerische 
Akademie der Medizinischen Wissenschaften; http://www. samw.ch/docs/Richtlinien/d_Forschungsunters.pdf.).

\section{Clinical outcome parameters}

Baseline demographic data were recorded as gender, age, American Society of Anaesthesiologists (ASA) score, elective or emergency procedure. Furthermore surgical expertise (RS vs. AS), duration of operation, length of hospital stay, 30-day perioperative morbidity and mortality were analysed. Bile duct injuries (Amsterdam criteria), number of vascular injuries, bowel or splenic lesions were considered as intraoperative complications.

\section{Statistical analysis}

Data are presented as median and range. Descriptive and univariate groupwise statistical analysis were performed using SigmaStat 3.11.0 (Systat Software, Richmond, CA, USA) with $p<0.05$ defined as statistically significant.

\section{Results}

\section{Study collective}

A total of 1,747 LC were performed during 11 years with a patients' median age of 55 years (16-94 years, 63.6\% females, Table 1). No primary open cholecystectomy was performed during the observation period. The majority of operations were performed by AS $(n=977 ; 56 \%)$. One thousand one hundred fifty-nine operations $(66 \%)$ were carried out as elective and 588 operations (34\%) as urgent procedures. Urgent operations were primarily performed by AS ( $n=360 ; 61 \%$ [AS], $p=0.001)$, whereas no significant differences existed in elective procedures $(47 \%$ [RS] vs. $53 \%$ [AS], $p=0.3$ ). Patients with body mass index (BMI) up to $29.9 \mathrm{~kg} / \mathrm{m}^{2}$ (BMI $<24.9 \mathrm{~kg} / \mathrm{m}^{2}-62 \%$ [AS] vs. $38 \%$ [RS], $p=0.001$; BMI 25 to $29.9 \mathrm{~kg} / \mathrm{m}^{2}-54 \%$ [AS] vs. $46 \%$ [RS], $p=0.3)$ and above $35 \mathrm{~kg} / \mathrm{m}^{2}(61 \%$ [AS] vs. $39 \%$ [RS], $p=0.09)$ were more frequently operated by AS. Whereas patients with BMI between 30 and $34.9 \mathrm{~kg} / \mathrm{m}^{2}$ were more often treated by RS $(54 \%[\mathrm{RS}]$ vs. $46 \%[\mathrm{AS}], p=0.001$, Table 1). The majority of patients treated during the observation period had low ASA scores I-II (Table 2). The proportion of low ASA scores was higher in patients treated by RS, and high ASA scores were predominant in patients treated by AS.

Intraoperative course

Overall duration of operation was 88 (25-245) min for RS vs. $75(30-190)$ min by AS ( $p=0.001)$. Elective operations were shorter when performed by AS than RS (70 (30-190) [AS] min vs. 85 (25-240) $\min$ [RS], $p=0.001)$, whereas urgent operations showed no statistically significant differences in duration of operation between the two groups (90 (30-245) $\mathrm{min}[\mathrm{RS}]$ vs. 85 (30-240) [AS] min, $p=0.3$; Fig. 2). Conversion to an open cholecystectomy for technical difficulties was performed in 24 patients $(1.4 \%)$ without statistical significant differences between RS and AS ([RS] $n=9(1.2 \%)$ vs. [AS] $n=15$ $(1.5 \%))$. Overall intraoperative complications such as bile duct injury, vascular injury, bowel injury or splenic injury were documented in $1.2 \%(n=21)$ and were independent of the surgeons status $(n=8 ; 1.0 \%[\mathrm{RS}]$ vs. $n=13 ; 1.3 \%$ [AS], $p=0.6$; Table 3 ). In case of bile duct injuries, there were only minor bile duct leaks (type A lesions) and no major leaks (type B lesions), strictures (type C lesions) or complete dissections (type D lesions). Vascular injuries of the cystic artery were resolved by additional clipping of the vessel. Bowel injuries due to, e.g. caustic lesions were laparoscopically sutured. Haemostasis in case of splenic lesions was achieved by using a hemostyptic gaze.

Table 1 Patient demographics

Percentages in parentheses are proportions of all patients treated. $p$ values refer to comparison of RS vs. AS $R S$ resident surgeon, $A S$ attending surgeon, $L C$ laparoscopic cholecystectomy

\begin{tabular}{|c|c|c|c|c|c|}
\hline & & All patients & Treated by RS & Treated by AS & $p$ value \\
\hline \multicolumn{2}{|c|}{ Number of patients } & 1,747 & $770(44 \%)$ & $977(56 \%)$ & 0.5 \\
\hline \multicolumn{2}{|l|}{ Female } & 1,111 & $490(44 \%)$ & $621(56 \%)$ & 0.9 \\
\hline \multicolumn{2}{|c|}{ Median age; range } & $55 ; 16-94$ & $52 ; 16-93$ & $58 ; 17-94$ & 0.03 \\
\hline \multicolumn{2}{|l|}{ Elective LC } & 1,159 & $542(47 \%)$ & $617(53 \%)$ & 0.3 \\
\hline \multicolumn{2}{|l|}{ Urgent LC } & 588 & $228(39 \%)$ & $360(61 \%)$ & 0.001 \\
\hline \multirow[t]{4}{*}{$\mathrm{BMI}$ in $\mathrm{kg} / \mathrm{m}^{2}$} & $<24.9$ & 539 & $206(38 \%)$ & $333(62 \%)$ & 0.001 \\
\hline & $25-29.9$ & 657 & $299(46 \%)$ & $358(54 \%)$ & 0.3 \\
\hline & $30-34.9$ & 334 & $181(54 \%)$ & $153(46 \%)$ & 0.001 \\
\hline & $>35$ & 217 & $84(39 \%)$ & $133(61 \%)$ & 0.09 \\
\hline
\end{tabular}


Table 2 Preoperative ASA scores

\begin{tabular}{lllll}
\hline & ASA I & ASA II & ASA III & ASA IV \\
\hline All patients & 583 & 934 & 213 & 17 \\
Treated by RS & $282(48 \%)$ & $411(44 \%)$ & $71(33 \%)$ & $6(35 \%)$ \\
Treated by AS & $301(52 \%)$ & $523(56 \%)$ & $142(67 \%)$ & $11(65 \%)$ \\
$p$ value & 0.3 & 0.001 & 0.0001 & 0.2 \\
\hline
\end{tabular}

Percentages reflect the proportion of patients of each ASA score

$A S A$ American Society of Anaesthesiologists (score), $R S$ resident surgeon, $A S$ attending surgeon

\section{Postoperative course}

Overall mortality was $0.6 \%$ in both groups $(n=4 ; 0.5 \%$ [RS] vs. $n=8 ; 0.8 \%[\mathrm{AS}], p=0.5)$ with myocardial infarction or heart failure as causes of death. There was a reduction in mortality rate from $1 \%$ between 1999 and 2004 to $0.3 \%$ between 2005 and 2009. Mortality was higher in urgent operations compared to elective cases (elective LC $n=6$; $0.5 \%$; urgent LC $n=6 ; 1.0 \%, p=0.2$ ) but independent of surgical expertise.

There was a trend towards shorter length of hospital stay in patients treated by RS as compared to those treated by AS (elective LC 4 (1-49) days [RS] vs. 5 (2-83) days [AS], $p=0.1$; urgent LC 4 (2-46) days [RS] vs. 5 (1-83) days [AS], $p=0.1$. Overall 30-day morbidity including intraoperative complications, wound infections, bile leak, intraabdominal abscess and reoperations was $5.2 \%$ with a lower rate in patients treated by RS $(3.8 \%[\mathrm{RS}]$ vs. $6.2 \%[\mathrm{AS}], p=$ $0.02)$. The comparison of urgent and elective LC showed a higher 30-day morbidity rate in urgent LC $(7.8 \%$ in urgent LC vs. $3.8 \%$ in elective LC, $p=0.03$ ).

Subgroup analysis showed that patients with ASA score III and IV were more frequently operated by AS when

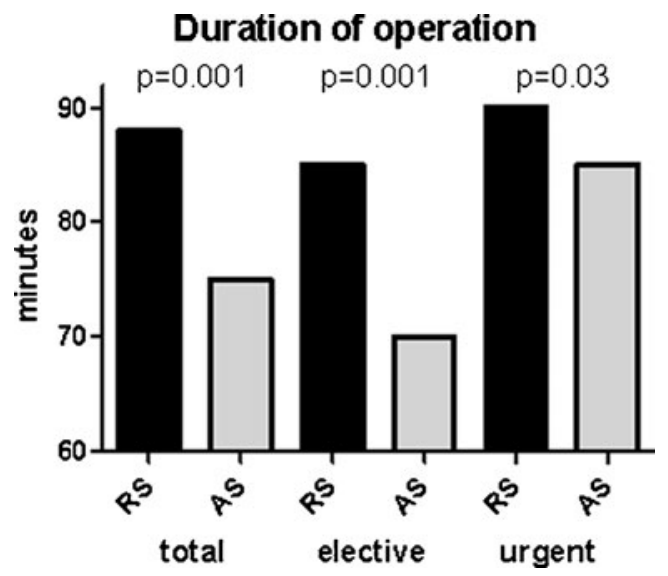

Fig. 2 Duration of operation in minutes of laparoscopic cholecystectomy in resident surgeons vs. attending surgeons in attention to operative priority priority was urgent, whereas patients with ASA score I/II and elective setting were more often treated by RS (Table 4). No significant differences were observed between patients treated by RS and AS regarding postoperative complications or mortality (Table 4). There were also no significant differences regarding the length of hospital stay of patients treated by RS or AS (Table 4).

\section{Discussion}

Our study shows that LC can be performed by RS with comparably low intraoperative and postoperative morbidity and mortality as by AS. As expected, the duration of operation was longer in cholecystectomies performed by RS, with this difference being greatest in elective operations. This fact is explained by the lower experience of RS in elective laparoscopic surgery before the operative time will decrease upon completion of the learning curve $[9,12,14]$.

Hobbs et al. [15] showed that intraoperative complications occurred in $1.37 \%$ of patients undergoing LC by a surgeon with an experience of one to 50 procedures compared to $0.8 \%$ complications by surgeons with an experience of more than 300 LC. Nevertheless in our study, there were no differences in intraoperative complications in LC performed by RS when compared to AS. This might be explained due to the fact that all operations performed by RS were instructed by experienced AS, thereby avoiding potential iatrogenic injury due to intraoperative misconception. In addition to that, the incidence of intraoperative complications was influenced by a bias in patient selection towards potentially "easier cases" for teaching operations and the surgical experiences of the RS as well. To provide a structured education and improve the technical skills of young surgeons, an appropriate instruction by experienced surgeons is mandatory. Likewise, the resident benefits by the presence of an experienced attending surgeon and frequently made technical mistakes during the learning curve can thereby be avoided $[5,10]$.

In our study emergent LC were predominantly performed by AS whereas elective procedures were performed 
Table 3 Intraoperative complications

\begin{tabular}{|c|c|c|c|c|c|c|c|c|c|}
\hline & \multicolumn{3}{|l|}{ Total } & \multicolumn{3}{|l|}{ Elective } & \multicolumn{3}{|l|}{ Urgent } \\
\hline & RS & AS & $p$ value & $\mathrm{RS}$ & AS & $p$ value & $\mathrm{RS}$ & AS & $p$ value \\
\hline Bile duct injury & $2(0.3 \%)$ & $3(0.3 \%)$ & 0.3 & $2(0.4 \%)$ & $1(0.2 \%)$ & 0.3 & 0 & $2(0.6 \%)$ & 0.4 \\
\hline Vascular injury & $3(0.4 \%)$ & $8(0.8 \%)$ & 0.1 & $1(0.2 \%)$ & $7(1.1 \%)$ & 0.05 & $2(0.8 \%)$ & $1(0.3 \%)$ & 0.3 \\
\hline Bowel injury & $3(0.4 \%)$ & $1(0.1 \%)$ & 0.2 & $3(0.6 \%)$ & 0 & 0.1 & 0 & $1(0.3 \%)$ & 0.6 \\
\hline Splenic injury & 0 & $1(0.1 \%)$ & 0.6 & 0 & $1(0.2 \%)$ & 0.5 & 0 & 0 & 1 \\
\hline
\end{tabular}

$R S$ resident surgeon, $A S$ attending surgeon, $L C$ laparoscopic cholecystectomy

similarly frequent by RS and AS. This might be explained due to clinical daily routine with anticipation of complications in patients in poor general condition (ASA score III and IV) and higher vulnerability of the gallbladder due to inflammatory reactions. Whereas patients undergoing an elective LC are mostly healthy with low ASA score and were therefore more frequently being performed by RS.

In general, postoperative complications in our study were more frequent after emergent LC than in elective operations which correspond to a previous report of 22,953 LC with about twofold higher risk of postoperative complications in patients with acute vs. chronic cholecystitis [2]. Compared to this study, our rate of postoperative complications with about $6.8 \%$ was lower than previously reported $12.2 \%$ [2]. Intraoperative complications such as bile duct injuries, distinct bleeding, bowel or splenic injuries were rare in both groups and showed no significant differences between RS and AS. The rate of bile duct injuries with $0.3 \%$ in our collective was comparable to other studies with $0.23 \%$ to $0.4 \%$ [11, 16]. Regarding the Amsterdam classification of bile duct lesions [17], all bile duct injuries in our series were type A lesions which could be treated by placing an additional clip during the initial operation and no further surgical intervention was necessary. There was a trend towards a higher rate of bleedings in case of operations performed by AS but without statistical differences in the outcome of these patients. This fact may be explained by the higher operative experience of AS and thereby potentially a lack of fear or respect of intraoperative iatrogenic bleeding complications.

Waage et al. [16] showed in a study of 152,776 cholecystectomies a significant decrease of bile duct injuries when intraoperative cholangiography was used. Hobbs et al. [15] could demonstrate that the risk for bile duct injuries was decreasing with surgeons experience and with the use of intraoperative cholangiography. Strasberg et al. [18] described in 1995 the technique of "critical view of safety" with dissecting the Calot's triangle free of fat and fibrous tissue in order to obtain a clear view on the cystic duct and artery. In our institution both techniques of intraoperative cholangiography and "critical view of safety" are performed and are responsible for the small rate of bile duct injuries. The value of intraoperative cholangiography has been extensively debated in the literature [15, 19-21]. Advantages of conducting routine cholangiography during $\mathrm{LC}$ are to retain the ability to perform this measure in cases

Table 4 Subgroup analysis of ASA score, urgent vs. elective laparoscopic cholecystectomy in resident surgeons (RS) vs. attending surgeons (AS) regarding hospital stay, postoperative complications and mortality

\begin{tabular}{|c|c|c|c|c|c|c|c|c|}
\hline & \multicolumn{4}{|c|}{ ASA score I/II } & \multicolumn{4}{|c|}{ ASA score III/IV } \\
\hline & \multicolumn{2}{|l|}{ Elective } & \multicolumn{2}{|l|}{ Urgent } & \multicolumn{2}{|l|}{ Elective } & \multicolumn{2}{|l|}{ Urgent } \\
\hline & RS & AS & RS & AS & RS & AS & $\mathrm{RS}$ & AS \\
\hline Number of patients $(\%)$ & $495(64 \%)$ & $537(55 \%)$ & $200(26 \%)$ & $287(29 \%)$ & $47(6 \%)$ & $80(8 \%)$ & $28(4 \%)$ & $73(8 \%)$ \\
\hline$p$ value & 0.0001 & & 0.1 & & 0.09 & & 0.0002 & \\
\hline Hospital stay in days (range) & $4(2-48)$ & $4(2-83)$ & $4(2-38)$ & $5(2-83)$ & $4.5(3-49)$ & $9(4-49)$ & $9(3-46)$ & $9(1-57)$ \\
\hline$p$ value & 0.1 & & 0.1 & & 0.08 & & 0.1 & \\
\hline Postoperative complications (\%) & $11(2 \%)$ & $18(3 \%)$ & $9(5 \%)$ & $11(4 \%)$ & $3(6 \%)$ & $13(16 \%)$ & $7(25 \%)$ & $19(26 \%)$ \\
\hline$p$ value & 0.08 & & 0.2 & & 0.06 & & 0.2 & \\
\hline Mortality (\%) & 0 & 0 & 0 & 0 & $1(2 \%)$ & $3(4 \%)$ & $3(11 \%)$ & $5(7 \%)$ \\
\hline$p$ value & 1.0 & & 1.0 & & 0.4 & & 0.2 & \\
\hline
\end{tabular}


of questionable anatomy or patency of extrahepatic bile ducts. In our opinion, apart from safety reasons, the use of intraoperative cholangiography is its value as an educational tool to improve laparoscopic skills, despite prolonging the operation for a few minutes and having to use $\mathrm{C}$-arm fluoroscopy.

In summary, we believe that LC is a safe procedure in the hands of RS-provided adequate supervision and when performed within a clearly defined teaching scenario including standardized operative steps. In contrast to other studies, we have actually found a higher rate of postoperative morbidity in LC performed by AS than by RS but with an overall low rate $[4,8,10-12]$. This fact may be explained by a selection bias of patients treated by AS with reduced general condition and higher ASA score.

Despite observing a decrease in overall mortality from $1 \%$ to $0.3 \%$ during the study period, our overall mortality of $0.6 \%$ is slightly higher than that of several previous reports (ranging from $0.3 \%$ to $0.5 \%$ ) $[2,22,23]$. Mortality was higher in urgent (1\%) than elective LC $(0.3 \%)$ and was to our surprise not associated with surgeons' experience. Furthermore, mortality in urgent LC decreased from $1.6 \%$ in 1999 to $0.6 \%$ in 2009 , a figure that compares well to a recent large Scottish study by Scollay et al., in which mortality in urgent LC dropped from $1.3 \%$ in 1997 to $0.55 \%$ in 2006 [24]. While it appears that gross mortality rates exceed today's standards, the authors believe that the subgroup of patients with high ASA scores require separate consideration: Most fatalities in our study were attributable to nonsurgical complications such as perioperative myocardial infarction or congestive heart failure typically seen in ASA score III and IV patients. Similar to our and the aforementioned Scottish trial [24], Murphy et al. [23] could demonstrate that advanced age, comorbidities and male gender are key factors associated with in-hospital complications in their analysis of 1,102,071 LC patients. In fact, Melloul et al. in their comparison of percutaneous drainage vs. emergency LC in acute cholecystitis in critically ill patients found mortality rates of $13 \%$ (drainage) and $16 \%$ (LC), highlighting the potential risks in this vulnerable group of patients [25]. By nature of our study design, no specific inferences may be drawn on the differences in perioperative risk in ASA score III/IV patients assigned to surgical vs. nonoperative treatment of acute cholecystitis. In fact, the authors believe that there is a need for future welldesigned prospective trials to provide better risk stratification for either treatment in patients with cholecystitis, especially elderly patients with higher ASA score.

The policy of our hospital is to avoid open cholecystectomy whenever technically feasible. During the observation period, there was actually not a single primary open cholecystectomy, and primary open cholecystectomies as part of other visceral surgery procedures such as liver resections, biliary tract resections due to cancer or pancreas resections (Whipple procedures) were excluded from analysis. The conversion rate of $1.4 \%$ was independent of the surgeon's experience and much lower than most reported rates in the literature with $5 \%$ to $19 \%[2,11,12$, $18,22,26]$, probably reflecting the motivation of the authors to stay with laparoscopy whenever justifiable. In contrast to our results, other authors actually reported higher rates of conversion in LC performed by AS compared to $\operatorname{RS}[12,27]$. Despite the low rate of conversion, there was not an increase of intraoperative complications seen in our study. All intraoperative complications which occurred were managed laparoscopically, and no conversion was necessary due to intraoperative complications. Because of the small number of intraoperative complications, statistical analysis revealed no differences on patient's outcome.

One third of our LC were performed as emergency procedures which is a high rate when compared to other studies such as the one by Giger et al. with $14.7 \%$ urgent LC [2]. This might be explained with our policy to perform LC during the initial admission whenever feasible. Our results of urgent $\mathrm{LC}$ as a teaching operation are comparable to a recently published study which showed that emergent LC can be performed safely by RS [27].

Surprisingly, the length of hospital stay was shorter in patients treated by RS compared to those treated by AS which is comparable with a previous report with shorter length of hospital stay in LC performed by RS with 1 vs. 2 days in patients treated by AS [27]. The difference of length in hospital stay in our study might be explained by patient selection and concomitant diseases of patients treated by AS, which is coherent to our findings concerning preoperative ASA scores and patient age.

In our study overall length of hospital stay of both groups was longer than compared to previous reports $[8$, 10]. This fact is likely not caused by the patient's general condition but may rather be a reflection of the Swiss healthcare system which is comparable to Germany [28, 29] or Austria [26]. Patients in some European countries, including Switzerland, receive longer inpatient treatment until they achieve a higher level of independence before being discharged home compared to the mostly American literature. At the same time, the costs of such longer hospital stays are covered by healthcare providers.

As limitations of this retrospective study should be noted, by nature, this study was performed in a retrospective manner, without randomization to either RS or AS treatment. By nature, more complex operations in "sicker" patients were performed by AS and the more "simple" cases performed by RS. This implies a selection bias in favour of the operative results achieved by RS. Furthermore, there was no individual differentiation between 
resident's or attending's surgical experience level. Also, it was not possible to differentiate operations that were only partly performed by RS, in which critical steps may have been performed by the AS present. The problem of nonrandomization pertains to any retrospective analysis of outcome registries, such as a recently published study by Raval et al. [30]. In their analysis of 607,683 surgical patients, a higher morbidity but equal or even lower mortality was observed in most subgroups of patients when operated by AS, which may again be explained by a potential selection bias in that RS on average tended to operate "simpler cases in healthier patients". Despite these limitations, we are convinced that our results reflect the real-life situation in a teaching hospital and emphasize the fact that equally good surgical outcomes may be achieved in patients operated by RS, and provided sound patient selection by the responsible AS is assured.

\section{Conclusion}

In conclusion, we report our experiences during a time period of 11 years of a series of laparoscopic cholecystectomies in a Swiss community hospital. We demonstrate that in face of adequate patient volume, appropriate teaching, selection and surveillance of surgical residents and adequate selection of patients feasible for resident laparoscopy training, laparoscopic cholecystectomies performed by residents are equally safe and associated with a morbidity and mortality as low as cholecystectomies performed by attending surgeons on the basis of short-term outcome parameters.

\section{Conflicts of interest None.}

\section{References}

1. Mühe E (1986) Die erste Cholezystektomie durch das Laparoskop. Langenbecks Arch Chir (Kongressband) 369:804

2. Giger UF, Michel JM, Opitz I, Inderbitzin DT, Kocher T, Krähenbühl L (2006) Risk factors for perioperative complications in patients undergoing laparoscopic cholecystectomy: analysis of 22,953 consecutive cases from the Swiss Association of Laparoscopic and Thoracoscopic Surgery database. J Am Coll Surg 203:723-728

3. Aggarwal R, Moorthy K, Darzi A (2004) Laparoscopic skills training and assessment. Br J Surg 91:1549-1558

4. Kanakala V, Bawa S, Gallagher P, Woodcock S, Attwood SE, Horgan LF, Seymour K (2010) Outcome of patients in laparoscopic training courses compared to standard patients. Surgeon 8:132-135

5. Aggarwal R, Darzi A (2006) Training in the operating theatre: is it safe? Thorax 61:278-279

6. Lee SL, Yaghoubian A (2010) Comparison of pediatric laparoscopic appendectomy outcomes between teaching and nonteaching hospitals: a multi-institutional study. J Laparoendosc Adv Surg Tech A 20:863-865
7. Balaa F, Moloo H, Poulin EC, Haggar F, Trottier DC, Boushey RP, Mamazza J (2007) Broad-based fellowships: a cornerstone of minimally invasive surgery education and dissemination. Surg Innov 14:205-210

8. Hwang CS, Pagano CR, Wichterman KA, Dunnington GL, Alfrey EJ (2008) Resident versus no resident: a single institutional study on operative complications, mortality, and costs. Surgery 144:339-344

9. Babineau TJ, Becker J, Gibbons G, Sentovich S, Hess D, Robertson S, Stone M (2004) The "cost" of operative training for surgical residents. Arch Surg 139:366-370

10. Koulas SG, Tsimoyiannis J, Koutsourelakis I, Zikos N, PappasGogos G, Siakas P, Tsimoyiannis EC (2006) Laparoscopic cholecystectomy performed by surgical trainees. JSLS 10:484-487

11. Lim HS, Salleh I, Poh BK, Tay KH (2005) Laparoscopic cholecystectomy: an audit of our training programme. ANZ J Surg 75:231-233

12. Kauvar DS, Braswell A, Brown BD, Harnisch M (2006) Influence of resident and attending surgeon seniority on operative performance in laparoscopic cholecystectomy. J Surg Res 132:159-163

13. Rageth JC (1998) Statistical models with reference to their value for medical process quality assurance. Schweiz Med Wochenschr 128:1703-1712

14. Hwang CS, Wichtermann KA, Alfrey EJ (2010) The cost of resident education. J Surg Res 163:18-23

15. Hobbs MS, Mai Q, Knuiman MW, Fletcher DR, Ridout SC (2006) Surgeon experience and trends in intraoperative complications in laparoscopic cholecystectomy. Br J Surg 93:844-853

16. Waage A, Nilsson M (2006) Iatrogenic bile duct injury: a population-based study of 152,776 cholecystectomies in the Swedish Inpatient Registry. Arch Surg 141:1207-1213

17. Bergmann JJGHM, van den Brink GR, Rauws EAJ, de Wit L, Obertop H, Huibregtse K, Tytgat GNJ, Gouma D-J (1996) Treatment of bile duct lesions after laparoscopic cholecystectomy. Gut 38:141-147

18. Strasberg SM, Hertl M, Soper NJ (1995) An analysis of the problem of biliary injury during laparoscopic cholecystectomy. J Am Coll Surg 180:101-125

19. Nickkholgh A, Soltaniyekta S, Kalbasi H (2006) Routine versus selective intraoperative cholangiography during laparoscopic cholecystectomy. Surg Endosc 20:868-874

20. Khan OA, Balaji S, Branagan G, Bennett DH, Davies N (2011) Randomized clinical trial of routine on-table cholangiography during laparoscopic cholecystectomy. Br J Surg 98:362-367

21. Hamad MA, Nada AA, Abdel-Atty MY, Kawashti AS (2011) Major biliary complications in 2,714 cases of laparoscopic cholecystectomy without intraoperative cholangiography: a multicenter retrospective study. Surg Endosc. doi:10.1007/s00464-011-1780-4

22. Harboe KM, Bardram L (2011) The quality of cholecystectomy in Denmark: outcome and risk factors of 20,307 patients from the national database. Surg Endosc 25:1630-1641

23. Murphy MM, Ng SC, Simons JP, Csikesz NG, Shah SA, Tseng JF (2010) Predictors of major complications after laparoscopic cholecystectomy: surgeon, hospital, or patient? J Am Coll Surg 211:73-80

24. Scollay JM, Mullen R, McPhillips G, Thompson AM (2011) Mortality associated with the treatment of gallstone disease: a 10-year contemporary national experience. World J Surg 35:643-647

25. Melloul E, Denys A, Demartines N, Calmes JM, Schöfer M (2011) Percutaneous drainage versus emergency cholecystectomy for the treatment of acute cholecystitis in critically ill patients: does it matter? World J Surg 35:826-833

26. Imhof M, Zacherl J, Rais A, Lipovac M, Jakesz R, Fuegger R (2002) Teaching laparoscopic cholecystectomy: do beginners 
adversely affect the outcome of the operation? Eur J Surg 168:470-474

27. Sanjay P, Moore J, Saffouri E, Ogston SA, Kulli C, Polignano FM, Tait IS (2010) Index laparoscopic cholecystectomy for acute admissions with cholelithiasis provides excellent training opportunities in emergency general surgery. Surgeon 8:127-131

28. Dotzenrath CM, Cupisti K, Raffel A, Aust B, Yang Q, Krüger B, Ohmann C, Röher HD, Goretzki PE (2005) Do Germans keep patients too long in hospital? A prospective randomized trial. World J Surg 29:1189-1193

29. Heistermann HP, Tobusch A, Palmes D (2006) Prevention of bile duct injuries after laparoscopic cholecystectomy. Zentralbl Chir 131:460-465

30. Raval MV, Wang X, Cohen ME, Ingraham AM, Bentrem DJ, Dimick JB, Flynn T, Hall BL, Ko CY (2011) The influence of resident involvement on surgical outcomes. J Am Coll Surg 212:889-898 